\title{
Integrating tuberculosis/HIV treatment: an evaluation of the tuberculosis outcomes of patients co-infected with tuberculosis and HIV in the Breede Valley subdistrict
}

Conradie H, Director, Ukwanda Centre for Rural Health, Associate Professor, Department of Family Medicine and Primary Care, Faculty of Medical and Health Sciences, University of Stellenbosch, Head of Unit, Department of Family Medicine, Worcester Hospital

Khati P, Research Assistant, Ukwanda Centre for Rural Health, Faculty of Medical and Health Sciences, University of Stellenbosch Pharoah H, Research Coordinator, Ukwanda Centre for Rural Health, Faculty of Medical and Health Science, University of Stellenbosch Adams S, Researcher, Ukwanda Centre for Rural Health, Faculty of Medical and Health Sciences, University of Stellenbosch Correspondence to: Hoffie Conradie, email hoffie@sun.ac.za

Keywords: tuberculosis/HIV treatment integration, human immunodeficiency virus, tuberculosis, outcomes

\begin{abstract}
Background: The Infectious Disease Clinic of Worcester Hospital introduced an integrated tuberculosis/human immunodeficiency virus (HIV) service in July 2009 to provide comprehensive management to patients who were co-infected with tuberculosis and HIV.
\end{abstract}

Method: In a retrospective cohort study that was carried out from 1 July 2009 to 31 March 2010, the tuberculosis outcomes of co-infected patients attending the Infectious Disease Clinic for antiretroviral (ARV) treatment and receiving their tuberculosis medication at the Infectious Disease Clinic, were compared with those of patients receiving ARV treatment at the Infectious Disease Clinic and tuberculosis treatment at their local clinic.

Results: Seventy-four per cent of patients completed their treatment and $26 \%$ were cured, with no defaults or deaths, in the tubercuolosis/HIV integrated cohort. Thirty-eight per cent completed their treatment, $45 \%$ were cured, $9 \%$ died and another $9 \%$ defaulted in the cohort receiving their tuberculosis treatment at a local clinic. This indicates that there was a significantly better tuberculosis outcome in the tuberculosis/HIV cohort ( $p$-value $<0.05)$.

Conclusion: The significantly better tuberculosis outcome that resulted when tuberculosis and HIV services were integrated led to services being integrated in the Breede Valley subdistrict.

(P) Peer reviewed. (Submitted: 2013-01-25, Accepted: 2013-04-04). @ SAAFP

SA Fam Pract 2013;55(5):478-479

\section{Introduction}

The Infectious Disease Clinic of Worcester Hospital provides antiretroviral (ARV) treatment to eligible patients who are infected with human immunodeficiency virus (HIV) in the Breede Valley subdistrict of the Cape Winelands District. Worcester Hospital is a regional hospital that also serves the Breede Valley subdistrict of the Cape Winelands District. Breede Valley subdistrict has a community day centre and six clinics. Historically, patients who attended the Infectious Disease Clinic and who had been diagnosed with tuberculosis were referred to their local clinic for tuberculosis treatment. In July 2009, an integrated tuberculosis/HIV service was established at the Infectious Disease Clinic with the assistance of the Ukwanda Centre for Rural Health to provide a comprehensive service that included screening for, and diagnosis and treatment of tuberculosis to patients infected with HIV and attending the Infectious Disease Clinic. Patients who were diagnosed with tuberculosis received their treatment at the Infectious Disease Clinic. Such treatment collaborations have demonstrated feasibility and cost-effectiveness. ${ }^{1}$

The aim of the study was to assess the impact that an integrated tuberculosis/HIV service had on the tuberculosis outcomes in patients who were HIV-positive and attending the Infectious Disease Clinic at Worcester Hospital. The objective was to compare the tuberculosis outcomes of patients who were co-infected with tuberculosis and HIV and attending the Infectious Disease Clinic for tuberculosis treatment, to those of patients who were co-infected with tuberculosis and HIV who continued to receive tuberculosis treatment at their local clinic in the Breede Valley subdistrict.

\section{Method}

The study population consisted of patients attending the Infectious Disease Clinic for ARV treatment who were diagnosed with tuberculosis between 1 July 2009 and 31 March 2010, either at the Infectious Disease Clinic or at their local clinic. A retrospective cohort study design was applied. The integrated clinic cohort consisted of patients receiving both ARV and tuberculosis treatment at the Infectious Disease Clinic when the tuberculosis/HIV integrated service was established. The second study cohort comprised patients receiving ARV treatment at the Infectious Disease Clinic, but tuberculosis treatment at their local clinic. The tuberculosis outcomes in both cohorts were compared. WHO definitions of tuberculosis 
Table I: Demographic information and tuberculosis outcomes

\begin{tabular}{|c|c|c|c|}
\hline Variables & $\begin{array}{c}\text { Integrated clinic } \\
\mathrm{n}(\%)\end{array}$ & $\begin{array}{l}\text { Local clinic } \\
\text { n (\%) }\end{array}$ & $\begin{array}{l}\text { Total } \\
\text { n (\%) }\end{array}$ \\
\hline Participating patients & $42(43 \%)$ & $56(57 \%)$ & $98(100 \%)$ \\
\hline \multicolumn{4}{|l|}{ Age groups (in years) ${ }^{*}$} \\
\hline $0-17$ & $5(12 \%)$ & $3(5 \%)$ & 8 (8\%) \\
\hline $18-35$ & $19(45 \%)$ & 29 (52\%) & $48(49 \%)$ \\
\hline $36-53$ & $16(38 \%)$ & $23(41 \%)$ & $39(40 \%)$ \\
\hline $54-65$ & $2(5 \%)$ & $1(2 \%)$ & $3(3 \%)$ \\
\hline Mean age & 33.1 & 34.1 & 33.7 \\
\hline \multicolumn{4}{|l|}{ Gender" } \\
\hline Men & $25(60 \%)$ & $19(34 \%)$ & $44(45 \%)$ \\
\hline Women & $17(40 \%)$ & $37(66 \%)$ & $54(55 \%)$ \\
\hline \multicolumn{4}{|l|}{ Outcomes ${ }^{* *}$} \\
\hline Completed & $31(74 \%)$ & $21(37 \%)$ & $52(53 \%)$ \\
\hline Cured & $11(26 \%)$ & $25(45 \%)$ & $36(37 \%)$ \\
\hline Died & $0(0 \%)$ & $5(9 \%)$ & $5(5 \%)$ \\
\hline Defaulted & $0(0 \%)$ & $5(9 \%)$ & $5(5 \%)$ \\
\hline
\end{tabular}

treatment outcomes were applied where "cured" refers to patients with sputum conversion and "completed" to patients who completed treatment but did not meet the criteria for cure or failure - this includes patient initially sputum negative and those with extra pulmonary TB. ${ }^{2}$

Data were retrieved from a database of patients with tuberculosis at the Department of Health Sub-District Office, as well as from the tuberculosis register at the Infectious Disease Clinic.

Data were captured using the SPSS ${ }^{\circledR}$ software programme. Chi-square analysis was applied to compare the two groups. Matching was not applied between the groups.

Permission to conduct the study was obtained from the Ethics Committee of Stellenbosch University, and subsequently from the Department of Health.

\section{Results}

A total of 98 patients participated in the study: 42 (43\%) in the tuberculosis/HIV integrated clinic and 56 (57\%) in the cohort who received their tuberculosis treatment at the local clinic. Patients were between the ages of two and 61 years. Almost $50 \%$ of co-infected patients were between the ages of 18 and 35 Fifty-five per cent of the patients were women and $45 \%$ men.

All patients in the integrated treatment group were either "cured" or "completed treatment", compared with 46 of 56 (82\%) in the group receiving TB treatment at the local clinic, this difference was statistically significant.

\section{Discussion}

The World Health Organization interim policy on collaborative tuberculosis/HIV activities recommends national tuberculosis and HIV coordination at regional, district and local level. ${ }^{3}$ Coetzee et al evaluated tuberculosis and HIV services in Khayelitsha, Cape Town, and also recommended the integration of these services. ${ }^{4}$ Friedland et al reported on the successful integration of tuberculosis/HIV services in Msinga, KwaZulu-Natal. ${ }^{5}$

While our retrospective study was limited by having unmatched groups, we confirmed higher treatment completion rates with an integrated service. Following this study, infectious disease services were decentralised and integrated ARV/TB management has now become standard at local clinic level in the Breede Valley subdistrict.

\section{Conclusion}

The results show that significantly better tuberculosis outcomes were achieved when tuberculosis/HIV services were integrated.

\section{References}

1. Sylla L, Bruce RD, Kamarulzama A, Altice FL. Integration and co-location of HIV/AIDS, tuberculosis and drug treatment services. Int J Drug Policy. 2007;18(4):306-12.

2. Tuberculosis. World Health Organisation. Available at: http://www.who. int/tb/publications/global_report/2008/table_a2_1/en/index1.html

3. Tuberculosis: interim policy on collaborative TB/HIV activities. World Health Organization [homepage on the Internet]. c2011. Available from: http://www.who.int/tuberculosis/hiv/faq/en

4. Coetzee D, Hilderbrand K, Goemaere E, et al. Integrating tuberculosis and HIV care in the primary care setting in South Africa. Trop Med Int Health. 2004;9(6):A11-A15.

5. Friedland G, Gandi N, Moll A, et al. Successful integration of tuberculosis and HIV treatment in rural South Africa: the Sizonq'oba study. J Acquire Immune Defic Syndr. 2009;50(1):37-43. 